\title{
Mitochondrial pseudogenes in the nuclear genome of Aedes aegypti mosquitoes: implications for past and future population genetic studies
}

\author{
Thaung Hlaing1,2, Willoughby Tun-Lin², Pradya Somboon ${ }^{3}$, Duong Socheat ${ }^{4}$, \\ To Setha ${ }^{4}$, Sein Min ${ }^{2}$, Moh Seng Chang 5 and Catherine Walton*1
}

\author{
Address: ${ }^{1}$ Faculty of Life Sciences, University of Manchester, Oxford Road, Manchester M13 9PT, UK, ${ }^{2}$ Medical Entomology Research Division \\ Department of Medical Research (Lower Myanmar), 5 Ziwaka Road, Dagon P.O., Yangon 11191, Myanmar, ${ }^{3}$ Department of Parasitology, Faculty \\ of Medicine, Chiang Mai University, Chiang Mai 50200, Thailand, ${ }^{4}$ National Centre for Malaria, Parasitology and Entomology, Phnom Penh, \\ Cambodia and ${ }^{5}$ WHO - Western Pacific Regional Office, Phnom Penh, Cambodia \\ Email: Thaung Hlaing - Thaung.hlaing@postgrad.manchester.ac.uk; Willoughby Tun-Lin - wtunlin@mptmail.net.mm; \\ Pradya Somboon - psomboon@mail.med.cmu.ac.th; Duong Socheat - socheatd@cnm.gov.kh; To Setha - to_setha@yahoo.com; \\ Sein Min -wtunlin@mptmail.net.mm; Moh Seng Chang - Changm@cam.wpro.who.int; \\ Catherine Walton* - catherine.walton@manchester.ac.uk \\ * Corresponding author
}

Published: 6 March 2009

BMC Genetics 2009, 10:11 doi:10.1186/1471-2156-10-11
Received: 6 October 2008

Accepted: 6 March 2009

This article is available from: http://www.biomedcentral.com/147I-2156/I0/II

(C) 2009 Hlaing et al; licensee BioMed Central Ltd.

This is an Open Access article distributed under the terms of the Creative Commons Attribution License (http://creativecommons.org/licenses/by/2.0), which permits unrestricted use, distribution, and reproduction in any medium, provided the original work is properly cited.

\begin{abstract}
Background: Mitochondrial DNA (mtDNA) is widely used in population genetic and phylogenetic studies in animals. However, such studies can generate misleading results if the species concerned contain nuclear copies of mtDNA (Numts) as these may amplify in addition to, or even instead of, the authentic target mtDNA. The aim of this study was to determine if Numts are present in Aedes aegypti mosquitoes, to characterise any Numts detected, and to assess the utility of using mtDNA for population genetics studies in this species.
\end{abstract}

Results: BLAST searches revealed large numbers of Numts in the Ae. aegypti nuclear genome on I 46 supercontigs. Although the majority are short $(80 \%<300$ bp), some Numts are almost full length mtDNA copies. These long Numts are not due to misassembly of the nuclear genome sequence as the Numt-nuclear genome junctions could be recovered by amplification and sequencing. Numt evolution appears to be a complex process in Ae. aegypti with ongoing genomic integration, fragmentation and mutation and the secondary movement of Numts within the nuclear genome.

The PCR amplification of the putative mtDNA nicotinamide adenine dinucleotide dehydrogenase subunit 4 (ND4) gene from 166 Southeast Asian Ae. aegypti mosquitoes generated a network with two highly divergent lineages (clade I and clade 2). Approximately I5\% of the ND4 sequences were a composite of those from each clade indicating Numt amplification in addition to, or instead of, mtDNA. Clade I was shown to be composed at least partially of Numts by the removal of clade I-specific bases from composite sequences following enrichment of the mtDNA. It is possible that all the clade I sequences in the network were Numts since the clade 2 sequences correspond to the known mitochondrial genome sequence and since all the individuals that produced clade I sequences were also found to contain clade $2 \mathrm{mtDNA}$-like sequences using clade 2-specific primers. However, either or both sets of clade sequences could have Numts since the BLAST 
searches revealed two long Numts that match clade 2 and one long Numt that matches clade I. The substantial numbers of mutations in cloned ND4 PCR products also suggest there are both recently-derived clade I and clade 2 Numt sequences.

Conclusion: We conclude that Numts are prevalent in Ae. aegypti and that it is difficult to distinguish mtDNA sequences due to the presence of recently formed Numts. Given this, future population genetic or phylogenetic studies in Ae. aegypti should use nuclear, rather than mtDNA, markers.

\section{Background}

Mitochondrial DNA (mtDNA) has been used extensively over the last three decades in population genetic and phylogenetic studies in a wide range of animals from Drosophila to humans (e.g. [1-4]). The advantages of mtDNA include its general lack of recombination that results in a single demographic history for the whole molecule, and high copy number which allows ease of amplification [5]. Further, the relatively high mutation rate of mtDNA generates correspondingly high levels of polymorphism and divergence [5]. This makes mtDNA particularly informative for the determination of genetic population structure and inference of population history within species as well as for deducing phylogenetic relationships between closely related species. MtDNA has been applied to many insect taxa including bees [6] and ants [7], in addition to medically important insects such as Anopheles [8-10] and Aedes mosquitoes $[11,12]$ where it is particularly important to estimate gene flow for vector control purposes $[13,14]$. Most recently, the use of mtDNA sequences has been proposed for several DNA bar-coding initiatives for taxonomic identification and biodiversity assessment [15].

However, mtDNA also has its disadvantages. MtDNA data can be misleading in population and phylogenetic studies as it is particularly prone to selective sweeps [16]; it can introgress with relative ease between species [17]; and its population dynamics may be driven by intracellular symbionts [18]. One problem that can be particularly difficult to detect is the presence of nuclear mitochondrial pseudogenes (Numts) $[19,20]$. Numts result from the translocation of mitochondrial sequences from the mitochondrial genome into the nuclear genome and, once integrated, these non-functional sequences accumulate mutations freely. The potential for Numt amplification in addition to, or even instead of, the authentic target mtDNA sequence can seriously confound population genetic and phylogenetic analyses (reviewed in [21,22]). For example, the mistaken inclusion of Numt sequences in an mtDNA phylogeny resulted in incorrect phylogenetic relationships being proposed within the South American bird genus Scytalopus $[23,24]$. The high prevalence of Numts in gorillas has also been problematic as they initially obscured the presence of two genetically divergent groups of gorillas which has important implications for understanding their evolutionary history and future conservation [25-27].

In 2001, over 82 different eukaryotes, including 20 insect species, were known to have Numts [22]. However, there have been numerous reports of Numts since then, for example, in gorillas [25], domestic cats [28], chickens [29], and several insects such as bees [30] and ants [31]. Although taxonomically widespread, there is substantial variation among species in Numt copy number [32]. Even within insects, Numt copy number varies greatly with high numbers in Tribolium flour beetles, honeybee and the brown mountain grasshopper [30,33], but few or none in Drosophila and Anopheles mosquitoes [32]. Although a positive correlation between Numt copy number and genome size is not clear cut [29,32], genome size may partially explain taxonomic variation in Numt copy number [22]. In this context it worth noting that the genome of Ae. aegypti is particularly large, $1.3 \mathrm{~Gb}$ [34]. Numt fragments tend to be small, with the vast majority (>90\%) less than $1 \mathrm{~kb}$, and often less than $100 \mathrm{bp}[30,32,35]$. The longest Numts have been found in species with high Numt copy number, such as humans, mouse and rice [32]. Controlling for size of mtDNA, the largest reported Numt to date is 14,654 bp in humans which corresponds to $~ 88 \%$ of the mitochondrial genome [36].

MtDNA has been widely used to study genetic population structure in Ae. aegypti mosquitoes, the organism of study here [37-41]. This mosquito is thought to have spread relatively recently from its ancestral home in Africa to the rest of the Tropics where they are important vectors of dengue and yellow fever [42,43]. An understanding of genetic population structure and gene flow in Ae. aegypti is therefore of particular importance as this information is required for vector control [44-46]. The presence of Numts in Ae. aegypti could therefore seriously confound the interpretation of previous population genetic studies in $A$ e. aegypti as well as restrict the use of mtDNA in future studies.

Numt presence can manifest itself in several ways including: PCR ghost bands; extra bands in restriction profiles; sequence ambiguities; and unexpected phylogenetic 
placement ([22] and references therein). In our mitochondrial sequences of this species we found a significant proportion of sequence ambiguities suggestive of the presence of Numts. In general, previous authors have not reported sequence ambiguities in Ae. aegypti. The study by Paduan and Ribolla [47] is one exception but here the sequence ambiguities were attributed to heteroplasmy (where wild type and mutant mitochondrial genomes coexist in a cell [48]). Another possible indication of the presence of Numts is the deep clade structure within many worldwide populations of Ae. aegypti. Two divergent mtDNA clades have been reported from: Mexico $[37,49]$; Thailand [38]; Venezuela [41]; Australia [50]; and the Americas [40]. The aims of this study are therefore to determine if Numts are present in Ae. aegypti mosquitoes, to characterise any Numts present, and to assess the utility of using mtDNA for population genetics studies in this species. Using a combined experimental and bioinformatics approach utilizing the recent genome sequence of $A e$. aegypti (available at http://aaegypti.vectorbase.org/ Genome/Home/) [34] we show here that Numts are prevalent in Ae. aegypti and that many previous studies have likely included Numt sequences in their mtDNA sequences. Finally, we discuss the implications of the use of mtDNA in future population genetic studies of this species.

\section{Results}

\section{Numt identification using BLAST searches}

Nucleotide BLAST searches of the Ae. aegypti genome sequence with each of the protein-coding genes from the mtDNA sequence of Ae. aegypti (GenBank accession number: EU352212) identified Numts on 135 different genomic supercontigs (using a cutoff $E$ value of $10^{-4}$ ) or 98 supercontigs (using a cutoff $E$ value of $10^{-14}$ ) (Figure 1 ). An additional 11 supercontigs were found to contain Numts derived from the tRNA and rRNA genes. Any overlap of supercontigs due to misassembly would result in fewer, but longer, Numts being detected. Assuming that overlap of the supercontig sequences is minimal, the number and size of Numts detected here should be reliable estimates. The Numts appeared to have originated from all over the mitochondrial genome, with longer genes typically having given rise to more Numts. Sequence identities of Numts with the mtDNA sequence ranged from $83 \%$ to $100 \%$. The shortest Numts found were $28 \mathrm{bp}$ for protein coding regions (e.g. a CO1 fragment on supercontig $1.695, E$ value $=3 \times 10^{-5}$ ) and $63 \mathrm{bp}$ for non-protein coding regions (tRNA-Arg and tRNA-Ala on supercontig $1.774, E$ value $=4 \times 10^{-25}$ ). The majority of Numts were less than 300 bp in length (Table 1).

Although the majority of Numts are small in length, the penultimate column in Figure 1 shows that 16 supercontigs contain Numts that span two or more genes. Of these,
Table I: Size distribution of Ae. aegypti Numts (protein coding, tRNA and rRNA) detected by BLAST searches

\begin{tabular}{lc}
\hline Size (bp) & Number of Numts \\
\hline $21-40$ & 23 \\
$41-80$ & 56 \\
$81-160$ & 38 \\
$|6|-320$ & 23 \\
$32 \mid-640$ & 7 \\
$64 \mid-1280$ & 5 \\
$|28|-2560$ & 8 \\
$256|-5| 20$ & 2 \\
$5|2|-10240$ & 4 \\
13,086 & 1 \\
15,455 & 1 \\
\hline
\end{tabular}

7 supercontigs have sequences corresponding to 6 or more contiguous mtDNA genes. The remaining supercontigs contain one to three smaller fragments of mtDNA-like sequence with each fragment spanning no more than three genes. For these longer Numts there appears to be some pattern across supercontigs in the genetic make up of the Numts. For example, supercontigs 1.348, 1.62 and 1.287 all contain a long Numt that spans the contiguous

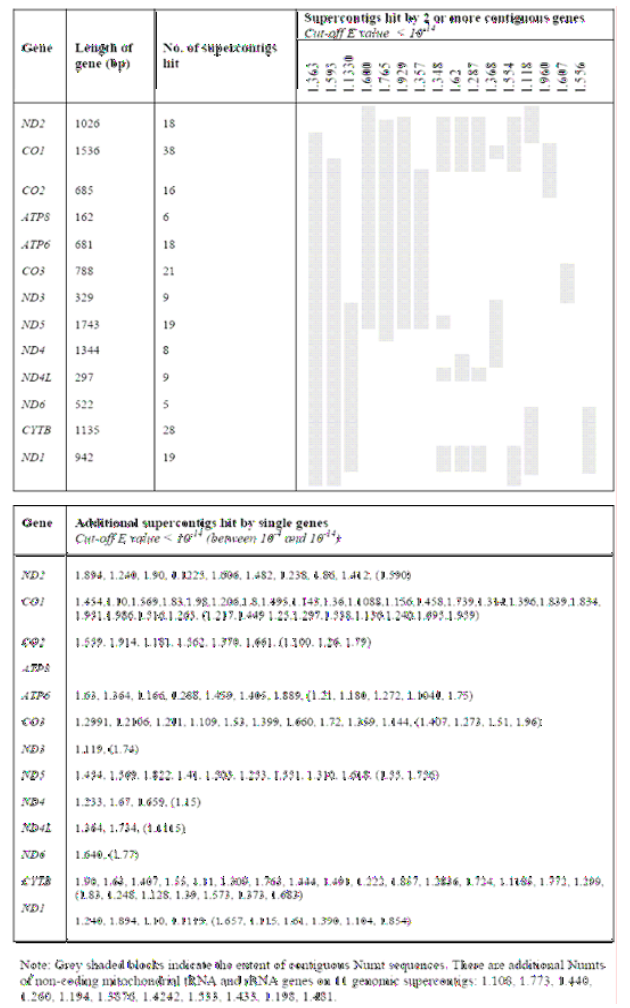

Figure I

Numbers and lengths of Numts of 13 protein-coding mitochondrial genes detected by BLAST searches of the Ae. aegypti genome sequence. 
genes ND1, ND2, and CO1 as well as part of the non-contiguous ND4L gene. In addition, supercontigs 1.600, 1.765 and 1.929 span the contiguous genes ND2, CO1, CO2, ATP8, ATP6, CO3, ND3 and ND5. With the exception of the first two, this set of contiguous genes is also contained by the Numt on supercontig 1.357. Although these contiguous genes were detected by BLAST using each protein coding gene individually, alignments between the mtDNA and the supercontigs showed that in all cases the Numts were contiguous across all coding and non-coding regions with $E$ values $<10^{-14}$. Despite their apparent similarity, the start and end positions of these long Numts differ in every case. There are also two supercontigs (1.363 and 1.593 ) that contain almost full length mtDNA copies.

It is possible that the long Numts identified by the BLAST search may not be true Numts but instead the result of misassembly during construction of this rather large and repetitive genome sequence. To address this question we investigate the detailed composition of the five longest putative Numts with the highest matches to the mtDNA sequence, which were those mostly likely to be due to misassembly. Figure 2 shows how these putative Numts differ from the mtDNA sequence. The two longest putative Numts on supercontigs 1.593 and 1.363 are almost full length and match almost completely with the mtDNA sequence. In supercontig 1.363 , the CO1 gene has the greatest number of mismatches with respect to the

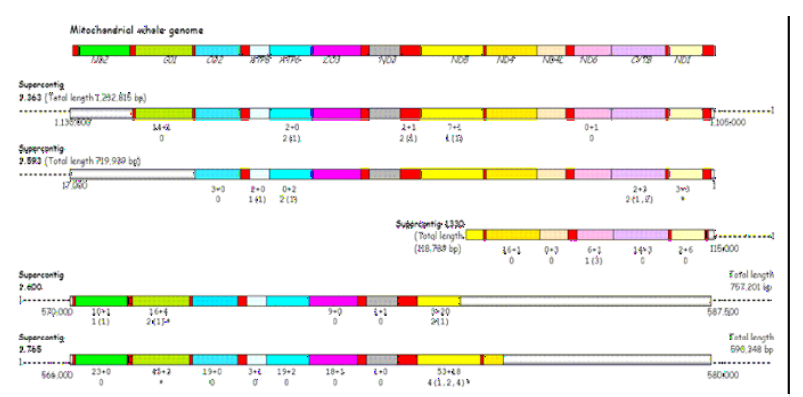

Figure 2

Alignment of five supercontigs containing the longest putative Numts with the mitochondrial genome. On the mitochondrial genome tRNA and rRNA genes are represented by red colour blocks and blocks of other colour indicate the coding genes as named. Corresponding coloured blocks on the supercontigs indicate regions of high homology with the mtDNA with mutational differences indicated below each gene as follows: Line I = numbers of synonymous + non-synonymous mutations, Line 2 = number of indels (length of indels in bp), * = Stop codons. Grey-shaded areas indicate regions of no homology and dotted lines represent the rest of the genomic supercontigs. The core haplotypes in clade 2 and clade I are identical to the corresponding ND4 regions on supercontigs $\mathrm{I} .363$ and $\mathrm{I} .593$, and supercontig I.I330, respectively.
mtDNA sequence, having one coding and 14 silent mutations. The ATPG, ND5 and ND6 genes and the six tRNAs between ND3 and ND5 have a small number of substitution mutations but there are no stop codons. All the indels are of a single base in simple sequence repeats of the same base so it is hard to exclude sequencing error. In the case of supercontig 1.363 the mtDNA-like sequence could therefore be due to genome sequence misassembly. Supercontig 1.593 makes a more convincing case for a Numt as although there are only a small number of silent and coding mutations (in the CO2, ATP8, ATP6, CYTB and ND1 genes) the indels are not all single base differences in simple sequence repeats and there is a stop codon in the ND1 gene. The placement of the mtDNA sequence at the end of supercontig 1.1330 (Figure 2) could be a sign of misassembly. However, this sequence contains no stop codons or frameshift mutations.

Supercontigs 1.600 and 1.765 have even greater numbers of substitutions compared to the mtDNA sequence and this coupled with the presence of stop codons is very clear evidence that these are Numt sequences. In all five of these long Numts the majority of substitutions are synonymous. This is not inconsistent with their being Numts as many of the observed substitutions may have occurred in the mtDNA rather than the Numt $[22,24]$ as, based on Drosophila, the mitochondrial mutation rate is likely to be 10 times higher than the nuclear mutation rate [51]. It is interesting to note that the gene fragments at the start of the Numt on supercontig 1.363, and at the starts and ends of the Numts on supercontigs 1.600 and 1.765, have much higher numbers of substitutions (indicated in Figure 2) with respect to the mtDNA sequence than the remaining genes in these Numts. This pattern is unlikely to indicate misassembly since it is found on Numts with both high and low levels of differentiation from the mitochondrial sequence. This pattern more likely indicates a heterogenous ancestry for these Numts.

\section{Recovered nuclear genomic sequences}

To further test the possibility of genome misassembly underlying the highly mt-DNA like sequences on Supercontigs 1.363, 1.593 and 1.1330, we designed and used primers to amplify genomic DNA fragments of 400-450 bp that span the transition between non-mtDNA-like sequence and the putative Numt (Table 2). (At the start of the Numt on supercontig 1.1330 and the end of the Numt on 1.593 there is little or no flanking regions (Figure 2) so these were not amplified.) Interestingly, primers designed to amplify across the Numt junction on supercontig 1.1330 did not recover the original sequence but instead a sequence (GenBank accession number: FJ463415) that differed from the original by 28 base substitutions and 5 indels of 1-4 bp distributed throughout the 412 bp amplified fragment. This sequence could not be found in 
Table 2: Primers used for mtDNA specific amplification and for amplifying across Numt junctions

\begin{tabular}{llll}
\hline Region amplified & Forward primer & Reverse primer & Fragment size (bp) \\
\hline Clade 2 specific ND4 gene region & ATGATAATTATACAATGAATTTTA & AACTCCCCCAATTAAGCTAATACTA & 282 \\
Start junction of Numt on 1.363 & GCTGGTGTGTGCATGAAACTAATC & TCGCGATTAAATGGCTGAAG & 406 \\
End junction of Numt on 1.363 & TCAAACGGACGAAGTTTGAGACAG & AAACCATGCCATTCCTTGAG & 444 \\
Start junction of Numt on 1.593 & ATAAGTGAGCCGAAGACACCGAG & GTCCGGTCTAGGGTCCTTTC & 413 \\
Start junction of Numt on I.1330 & TCACAATCACAGCCACTTTTCC & CCTATTCAAAACAGGTTTCGTTCAAG & 412 \\
\hline
\end{tabular}

the Ae. aegypti genome sequence by a BLAST search. Instead, this BLAST search identifed another similar sequence of $200 \mathrm{bp}$ on supercontig 1.43 ( $E$ value $5 \times 10^{-}$ ${ }^{72}$ ) that differed from the query sequence by 12 base substitutions throughout. Rather than indicating that supercontig 1.1330 contains missassembled mtDNA sequence, the finding of similar but different junction sequences may instead indicate there are sets of related Numts. The sequences of the Numt junctions at the starts and ends of the Numt on supercontig 1.363 and the start of the Numt on 1.593 (GenBank accession numbers: FJ463412FJ463414) fully recovered the database genome sequences. Overall, the sequence data on Numt junctions indicate that the long mtDNA-like sequences on these supercontigs are true Numts rather than the result of genome misassembly.

\section{Deep clade structure of putative mitochondrial haplotypes} To determine if Numts may be a problem in mtDNAbased population genetic studies of Ae. aegypti, we amplified and sequenced the putative mitochondrial gene nicotinamide adenine dinucleotide dehydrogenase (NADH) subunit 4 (ND4) from natural populations of Ae. aegypti. A total of 763 usable bases of the ND4 gene was generated from 166 Ae. aegypti mosquito individuals collected from Myanmar, Thailand and Cambodia. Of these, 141 sequences were unambiguous and corresponded to 38 unique haplotypes (GenBank accession numbers: FJ428759-FJ428796) varying in frequency from 1 to 50 . In a median-joining (MJ) network, these haplotypes fell into two divergent clades with both clades containing individuals from all three countries (Figure 3). The core sequences in each clade were separated by 17 mutations, 16 of which were synonymous changes. The one non-synonymous mutation was a conservative amino acid change from Ile to Val. The A+T content of the clade 1 and clade 2 core haplotypes was $76.4 \%$ and $75.6 \%$ respectively, typical of insect mtDNA. There were no frameshift or stop codon mutations within or between the two clades. Within clade 1 there were four non-synonymous mutations and within clade 2, two non-synonymous mutations. Clade 1 and clade 2 have somewhat similar topologies with a high frequency core haplotype. However, clade 1 has a more star-like structure with many individuals differing from the core by 1-4 mutations whereas in clade 2 several haplotypes are quite divergent from the core.

In addition to the 141 clear sequences we also obtained 25 ambiguous sequences, corresponding to $15 \%$ of the total sample. The ambiguous sequences possessed double peaks at 9-17 sites all of which were sites that differentiated the two clades. Since the forward primer used here differed from those used in previous studies [41] we repeated the amplification and sequencing of 12 individuals with ambiguous sequences using the orginal primers that amplified a shorter 359 bp fragment corresponding to the 3' end of our amplicon. Even for these shorter fragments, the ambiguities were consistently present, in the same individuals and involving the same sites that differed between the two clades. This situation was not changed even by redesign of the reverse primers. These

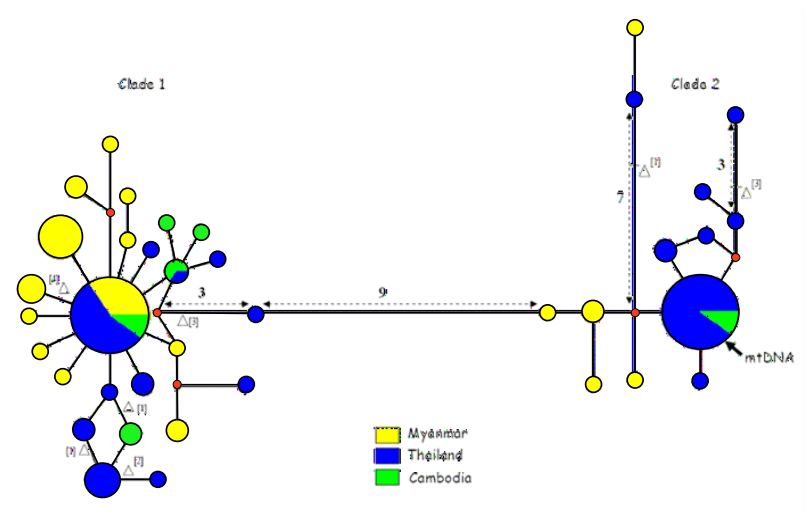

Figure 3

Median-joining (MJ) network for ND4 putative mtDNA sequences from I 4 I individuals of Ae. aegypti from Southeast Asia. Note: Each circle represents a unique haplotype. Circle size is propotional to haplotype frequency and circle colour indicates the country of origin. The lengths of branches between haplotypes are proportional to the number of mutations. Numbers along branches indicate 3 or more mutational differences between the haplotypes. $\Delta$ refers to non-synonymous mutations with amino acid changes: [I] Valine to Isoleucine; [2] Proline to Serine; [3] Isoleucine to Valine; [4] Serine to Proline. As indicated by an arrow, the published mtDNA sequence corresponds to the clade 2 core sequence. 
sequence ambiguities indicate that Numts are being amplified instead of, or together with, Ae. aegypti mtDNA. The core sequence of clade 2 has a 100\% sequence identity with the mtDNA sequence of Ae. aegypti (GenBank accession number: EU352212) which indicates that clade 1 most likely comprises Numts.

\section{Confirmation that clade I contains Numt sequences} Mitochondrial DNA was enriched from five fresh individuals from Chiang Mai, Thailand. The ND4 gene region was amplified and sequenced from both the total genomic DNA (prior to purification) and the enriched mtDNA for all individuals. The core clade 2 sequence was obtained for three individuals for both the total genomic DNA and the enriched mtDNA. However, the total genomic DNA of the remaining two individuals yielded ambiguous sequences that were composites of a clade 1 sequence and the clade 2 core sequence. Following enrichment, the mtDNA fraction generated a clear clade 2 sequence (Figure 4). In addition to confirming the sequence of the mtDNA, this demonstrates that the clade 1 sequences in these two individuals are due to Numts.

If all the clade 1 sequences obtained from the Southeast Asian mosquitoes were Numts we would also expect an mtDNA sequence to be present in each individual. We therefore developed primers that amplify 282 bp of the

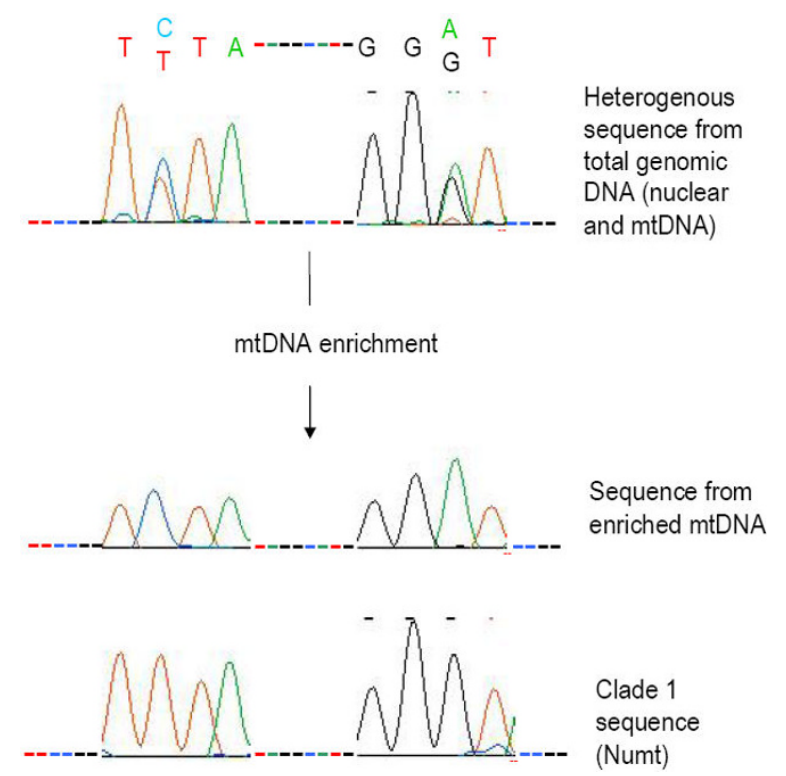

Figure 4

Example of the removal of a clade I Numt sequence from a heterogenous sequence by mitochondrial enrichment. The electropherogram profile shows superimposed peaks at positions 159 and 194 of ND4 heterogenous sequences. The same pattern is found in the complementary strand (data not shown).
ND4 gene from clade 2 (i.e. known mtDNA-like) sequences only (Table 2 ). These clade $2 / \mathrm{mtDNA}$-specific primers had 3 ' termini at bases that distinguished clade 2 from clade 1 . The PCR conditions under which these primers were specific for the clade 2/mtDNA sequence were determined using clade 1 and clade 2 PCR products as templates. Under these conditions the mtDNA-specific primers generated PCR products from all individuals that had previously given rise to unambiguous clade 1 sequences using the original primers. Nine of these amplicons were sequenced and all sequences were confirmed to fall into clade 2. For sequences of both clades to be present within an individual, at least one of the sequences must be a Numt. Based on the results of the mtDNA enrichment which showed clade 1 Numts and that the known mtDNA sequence corresponds to clade 2, the most parsimonious explanation is that all the clade 1 sequences are Numts. However, we cannot exclude the possibility that the clade $2 / \mathrm{mtDNA}$ specific primers are amplifying clade 2-like Numts such as those detected on supercontigs 1.593 and 1.363 by the BLAST searches.

\section{Sequences from cloned PCR products}

To further assess the extent of Numt presence in Ae. aegypti we also cloned and sequenced PCR products of the ND4 gene from two individuals from which we had obtained ambiguous sequences. We expected Numts to be characterised by being present as multiple, not necessarily closely related, sequences within an individual and with the possible presence of stop codon and/or frameshift mutations. Mitochondrial genes on the other hand should be comprised primarily of one sequence with the possible existence of sequence variants differing by one (or at most two) mutations due to Taq error during amplification captured by the cloning process, assuming an error rate of $7.3 \times 10^{-5}$ per bp per duplication [52]. This logic was also used by Arctander [24] to distinguish a Numt and mitochondrial sequence. However, contrary to our expectation, we found that for both individuals each clade contained multiple, divergent cloned sequences with around one third of the mutations being amino acid changes (Figure 5). Those sequences which cluster within a few mutations of the sequence inferred by direct sequencing of the corresponding original PCR product could just be the result of Taq error. However, even if the Taq error rate was higher than predicted, there are at least four outlier sequences that differ by 7-9 mutations from the original PCR-based sequence which cannot be attributed to Taq error alone. For example, in Clade 2 one haplotype differs from the putative mtDNA sequence in that individual by seven mutations including one non-synonymous substitution and one stop codon. These divergent sequences are most likely Numt sequences, indicating that there are both recently derived clade 1 - and clade 2-like Numts. These novel variants of the ND4 gene could not be 


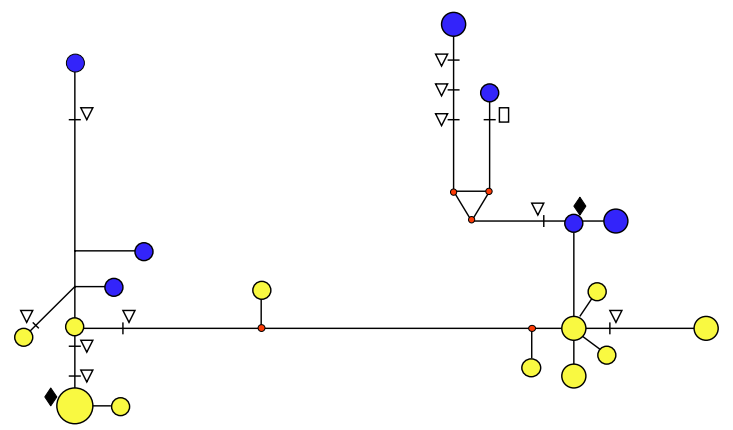

Figure 5

Median-joining network showing mutational differences among clones of ND4 sequences from two mosquito individuals. Haplotype colour indicates which of the two individuals a sequence originated from. Symbols denote the following: $€$ stop codon; $\nabla$ amino acid change; $\checkmark$ core clade I and core clade 2 haplotypes from Figure 3.

found in the genome sequence by BLAST searches. This may simply be because the Southeast Asian individuals we sequenced differ in genomic composition from the sequenced strain or they may be revealed in the future when a more complete assembled version of the genome is released.

\section{Discussion}

High numbers of Numts in the Ae. aegypti nuclear genome The results of the BLAST searches demonstrate the presence of high numbers of Numts in the genome of $A e$. aegypti with Numts detected on a total of 146 different supercontigs. This is comparable to other insects that are considered to have large numbers of Numts including the honeybee, Apis mellifera, and the Tribolium flour beetle which have Numts at an estimated 575 and 57 genomic locations, respectively [30]. It is notable that other dipterans studied to date, D. melanogaster and An. gambiae, which have few or no Numts, have much smaller genomes than Ae. aegypti; $176 \mathrm{Mb}$ and $278 \mathrm{Mb}$ compared to $1.3 \mathrm{~Gb}$ for Ae. aegypti. Almost half the genome of Ae. aegypti is composed of transposable elements [34] which may indicate that this species is generally unable to rid itself of non functional DNA, including Numts.

In agreement with studies in other taxa [e.g. [30]], the majority of the Numts detected here were short. However, we also noted many long Numts with six being greater than $7 \mathrm{~kb}$ in length with the two longest on supercontigs 1.363 and 1.593 (Figure 1). Although these latter two differed very little from the mitochondrial sequence they appear to be real rather than the result of misassembly as there are some frameshift and stop mutations (albeit very few in number) and the presence of Numt-genome junction points was confirmed by amplification and sequenc- ing. At $15.46 \mathrm{~kb}$ and $13.07 \mathrm{~kb}$, the Numts on supercontigs 1.363 and 1.593, respectively, are amongst the longest reported to date. Other species with long Numts are humans [36], Arabidopsis, rice, house mice [32], domestic cats [19], and voles [53] that have Numts of $14.65 \mathrm{~kb}$, $20.13 \mathrm{~kb}, 13.32 \mathrm{~kb}, 12.4 \mathrm{~kb}, 7.9 \mathrm{~kb}$ and $4 \mathrm{~kb}$ in length, respectively.

In general, as the Numts in Ae. aegypti get progressively shorter in length their similarity to the mitochondrial sequence decreases (Figure 2). This is consistent with the repeated movement of long mtDNA sequences to the nucleus which then decay through time becoming both fragmented and mutated. This is also consistent with our observation that, despite some variation among genes, all the mitochondrial genes are represented in Numts in our study. This is similar to the situation in humans $[36,54]$ and mouse and Ciona [32] but contrasts with several other studies which have noted a very marked preference for particular mtDNA genes to become Numts [29,30]. The situation in which the Numt on supercontig 1.1330 was found to have a homologous Numt with the same end point but slightly different genomic flanking sequence has been found previously [54]. Such Numt copies could have arisen from the secondary integration of Numts from one nuclear genomic location to another [28,36,55]. Genomic rearrangements involving Numts seem common [36]. In accord with this, the pattern seen here of high substitution rates at the extreme ends of three of the long Numts indicates that these Numts could have been formed by the homologous recombination of long mtDNA sequences into genome positions that already contained Numts. However, it is also difficult to exclude the possibility of genome misassembly in giving rise to this pattern. Overall, our data lead to the conclusion that Numt evolution in $A e$. aegypti is a very complex and dynamic process.

\section{Origin of Clade I and Clade 2 type Numts}

The genome sequence was obtained from a highly inbred strain of $A e$. aegypti [34]. It seems unfeasible that this strain could have maintained two mtDNA sequences, corresponding to clades 1 and 2, through successive bottlenecked generations. The evidence presented here demonstrates clearly that at least some, and possibly all, clade 1 sequences originate from Numts and not from mtDNA. Not only do the clade 1 sequences generated from ND4 PCR products (Figure 3) correspond to the sequences removed by mtDNA enrichment and the Numt on supercontig 1.1330 , but clade 2 sequences were also found in all individuals that generated clade 1 sequences. The clade 2 sequences generated from ND4 PCR products (Figure 3) correspond to the reported mtDNA sequence. However, it is possible that not all of these clade 2 sequences have arisen from mtDNA as the data indicate that there are also very recently derived Numts: firstly, the 
putative Numts on supercontigs 1.363 and 1.593 are almost identical to the mtDNA sequences; and secondly, the clade 2 sequences of cloned PCR products have high numbers of mutations (Figure 5). Although it could be argued that these findings are the result of misassembly of the nuclear genome sequence and high Taq error rate, respectively, these do not appear to be the most parsimonious arguments.

The sites that differ between our clade 1 and clade 2 sequences are the same as those found in a large number of other studies [37-41,56] using the putative ND4 mtDNA gene. In this and previous studies, the primers used were designed using mtDNA sequences from related taxa (Ae. albopictus). It has been noted that this strategy is inherently prone to the co-amplifcation of Numts [57]. Many of the previous studies e.g. [37-39,41] relied on single-strand conformation polymorphism (SSCP) coupled with sequencing of a few individuals. If co-amplifying Numt sequences were present and formed additional bands on the gene they may have been disregarded, as SSCP gels can contain background bands due to the same sequence adopting alternative conformations. This may explain why, besides this one, there has only been one other report [47] of heterogenous sequences using this marker. In this latter case the authors attributed the heterogenous sequences to heteroplasmy because the mixed sequences contained the variation found in their clade 1 and clade 2 sequences which they believed to all be true mtDNA.

There are at least two possible explanations for the origin of the clade 1 and clade 2 type Numts. Firstly, the Numts could have originated from the same species at different time points with the clade 1 sequences being entirely due to Numts and transferring at an earlier time. The deep clade structure would therefore have been formed by the movement of clade-1 like mtDNA into the nuclear genome, presumably in Africa of the order of one million years ago (making the assumption that divergence rates will have been similar to those of mtDNA estimated at $2.3 \%$ per million years [58]). This might at first appear inconsistent with the lack of amplification of other recently derived Numts, particularly of sequences intermediate between clades 1 and 2 that might be expected from continual mtDNA transfers to the nucleus. However, the ease with which clade 1 Numts are amplified could be explained by their being present in multiple copies in the genome, for example, due to secondary integration as suggested above. The one coding and 16 silent substitutions separating clade 1 and clade 2 sequences indicate the action of purifying selection. These substitutions would therefore have accumulated primarily along the clade 2 mtDNA lineage. This is consistent with the clade 1 sequences being multi-copy Numts as their independ- ently acquired substitutions would not be apparent in sequenced PCR products. The star-like structure of the clade 1 sequences in the haplotype network is, however, hard to explain under this scenario.

A second possible explanation is that both clade 1 and clade 2 Numts are of recent origin but have originated from different source populations/species with divergent mtDNAs. A likely (but not only) candidate for a source of divergent mtDNA is the forest form of Ae. aegypti from Africa. The domestic form of Ae. aegypti studied here derives from Africa where there is known to be ongoing gene flow between it and the forest form [43]. Putative mtDNA ND4 haplotypes of the forest form are shared with those of the domestic form in West Africa [59] and (from a comparison with our sequences) these comprise both clade 1 and clade 2 sequences. However, it is possible that the forest and domestic form have recently made secondary contact following a period of allopatry during which their mtDNA could have diverged. Clade 1 and clade 2 Numts could have arisen pre or post secondary contact and in either the forest and/or the domestic form.

These alternative hypotheses for the origin of the clade 1 and clade 2 Numts (single source at different times versus different sources and recent origin) could be distinguished by sequencing enriched mtDNA from a large number of individuals, ideally including those of the forest form. Under the second hypothesis at least some individuals should have clade 1 type mtDNA. This should be performed in conjunction with amplifications of the total genomic DNA using clade specific primers to determine if clade 1 or clade 2 Numt sequences are present.

\section{Implications for inference of population history and genetic structure}

The unknowing inclusion of Numts sequences in mtDNA based analyses could lead to mistaken inferences of population structure and population history. Since Ae. aegypti mosquitoes have spread from Africa to the rest of the Tropics relatively recently, the deep clade structure found in previous studies has often been interpreted to suggest colonization from at least two different source populations (and/or by many individuals) [38,40,46,60,61]. However, the deep clade structure could instead be due to the presence of Numts. In this case, the two deep clades could also mistakenly be interpreted to indicate the presence of two distinct species outside of Africa. This exemplifies the problem pointed out by Song et al. [62] that the use of mtDNA genes for DNA barcoding could lead to the overestimation of species numbers.

Although the putative mtDNA sequences can clearly detect genetic population structure among regional populations of Ae. aegypti (Table 3), the reliability of differenti- 
Table 3: Population pairwise FST values estimated using clade I and clade 2 sequences

\begin{tabular}{|c|c|c|c|c|}
\hline Sample locations & Sequences used & Myanmar (Yangon) & $\begin{array}{l}\text { northwest Thailand } \\
\text { (Chiang Mai) }\end{array}$ & $\begin{array}{l}\text { northeast Thailand } \\
\text { (Ubon Ratchathani) }\end{array}$ \\
\hline \multirow{2}{*}{$\begin{array}{l}\text { northwest Thailand } \\
\text { (Chiang Mai) }\end{array}$} & Clade 2 (mtDNA-like) & $0.323^{*}$ & & \\
\hline & Clade I & $0.248^{*}$ & & \\
\hline \multirow{2}{*}{$\begin{array}{l}\text { northeast Thailand } \\
\text { (Ubon Ratchathani) }\end{array}$} & Clade 2 (mtDNA-like) & 0.082 & 0.088 & \\
\hline & Clade I & 0.043 & $0.282 *$ & \\
\hline \multirow[t]{2}{*}{ Cambodia (Battambang) } & Clade 2 (mtDNA-like) & 0.021 & 0.110 & -0.105 \\
\hline & Clade I & 0.136 & $0.152 *$ & $0.136 *$ \\
\hline
\end{tabular}

* Significant $(P<0.01)$

ation estimates would be affected by the inclusion of Numts. This is particularly the case if the proportion of Numt amplification is not consistent across populations. This is suggested here by the notably lower proportion of clade 1 amplification from the northwestern Thai population $\left(\chi^{2}=22.02, \mathrm{df}=3, P<0.001\right)$. If the clade 1 sequences are actually Numts, this results in a dramatic increase of the apparent level of differentiation between the northwest and eastern Thai populations (from FST of 0.088 to 0.28 ; Table 3 ).

There are a number of means by which Numt co-amplification can be limited such as mitochondrial purification before DNA extraction, long PCR, reverse transcriptase PCR, or using tissue rich in mtDNA (e.g. muscle) but none are guaranteed to absolutely overcome the problem ([22] and references therein). Perhaps the simplest solution to the problem providing that the Numts are monophyletic with respect to the mtDNA sequences, is their removal by restriction enzyme digestion or their avoidance by the use of mtDNA-specific primers [22]. However, these possible solutions will not work here if, as the BLAST results and sequences of clones indicate, there are very recently derived Numt sequences. We therefore advocate that future studies of population structure in Ae. aegypti use other markers. With the genome sequence available there are a large number of nuclear markers to choose from.

\section{Conclusion}

Using a variety of approaches we conclude that Numts are prevalent in Ae. aegypti and that Numt evolution in Ae. aegypti is a very complex and dynamic process. These data also show that the deep clade structure we and others have been obtaining when performing supposedly mtDNAbased studies in Ae. aegypti may in fact be due to Numts. Some interpretations of the population and colonisation history of Ae. aegypti made to date may therefore be unreliable. We advocate that future studies of genetic population structure and gene flow in Ae. aegypti avoid using mtDNA data and use nuclear markers instead.

\section{Methods}

\section{BLASTN searches of Ae. aegypti genome sequence}

This study utilized the genomic sequence of Ae. aegypti (version-AaegL1) sequenced from the Liverpool LVP strain to approximately $8 \times$ coverage by the Broad Institute and The Institute for Genomic Research (TIGR). The genome sequence has over 1.31 billion bp and comprises 4,758 supercontigs but these have not yet been physically mapped to chromosomes http://aaegypti.vectorbase.org/ Genome/Home/.

This genomic sequence was searched for Numts by BLASTN [63] on VectorBase for each of the 13 proteincoding genes, 22 tRNA genes and 2 rRNA genes from the Ae. aegypti mitochondrial genome sequence of $16,655 \mathrm{bp}$ (GenBank accession number: EU352212). Threshold levels for the inference of Numts from BLASTN hits were taken as expectation values ( $E$ values) of $10^{-4}$ or $10^{-14}$, as used in other studies $[30,32,64]$. We determined the extent to which the hits against single genes were contiguous and due to single large Numts by carrying out alignments of the mtDNA genome with the regions of supercontigs containing multiple hits using ClustalX $(1.83)[65,66]$. The aligned sequences were visualized in MEGA ver. 4 [67] to determine the lengths of Numt sequences and to identify the Numt-supercontig junction points. The same software was also used for the translation of protein coding sequences to identify missense mutations and stop codons.

\section{Extraction of genomic DNA, PCR amplication and direct sequencing}

DNA was extracted from individual mosquitoes using a standard phenol/chloroform method [68]. The final DNA pellet was suspended in $20 \mu \mathrm{l}$ water $\left(\mathrm{dH}_{2} \mathrm{O}\right)$ and was diluted 1:20 to make a working solution. A fragment of the nicotinamide adenine dinucleotide dehydrogenase (NADH) subunit 4 mitochondrial (ND4) gene was amplified using the primers: ND4F (5'-GTTTAGATATARTTTCTTAYGG-3') and ND4R (5'-CTTCGDCTTCCWADWCGTTC- 
3'). The ND4R primer was the same as that used in previous studies in Mexico [37,49], Thailand [38] and Venezuela [41]. The ND4F primer used here was designed in Primer3 ver. 0.4.0 [69] from an alignment of the ND4 region of the mtDNA of Aedes albopictus (GenBank accession number: AY072044), Anopheles gambiae (GenBank accession number: L20934) and Anopheles quadrimaculatus (GenBank accession number: L04272). The combination of primers used here therefore amplified a fragment that had the same 3 ' end as that of the previous studies but which was longer, being 763 bp rather than 359 bp in length.

The concentrations of the PCR reactants were $1 \times$ ammonium buffer (CLP, Northampton, UK), $2.5 \mathrm{mM} \mathrm{MgCl}_{2}$, $200 \mu \mathrm{M} d \mathrm{dNT}$ and $0.4 \mu \mathrm{M}$ primers. Amplifications were carried out in $50 \mu \mathrm{l}$ volumes using $1 \mu \mathrm{l}$ of template DNA. The PCR programme was modified from the previous Thailand and Mexico studies $[37,38,49]$. In an intial hot start, the reagents were heated to $95^{\circ} \mathrm{C}$ for $2 \mathrm{mins}$ prior to the addition of 1.25 units of Thermoprime plus DNA polymerase (CLP, Northampton, UK). This was followed by 38 amplification cycles consisting of $30 \mathrm{sec}$ at $92^{\circ} \mathrm{C}, 1$ min at $50^{\circ} \mathrm{C}$ and $40 \mathrm{sec}$ at $72^{\circ} \mathrm{C}$, followed by a final extension for 5 mins at $72^{\circ} \mathrm{C}$. PCR reactions were carried out on a GeneAmp ${ }^{\circledast}$ PCR System 9700 thermocycler (Applied Biosystems, Warrington, UK). PCR products were purified using Montage columns (Millipore, Billerica, MA, USA) and sequenced in both directions (Macrogen Inc., Seoul, Korea). DNA sequences were assembled using the Sequencher multiple sequence editor program ver. 4.5 (Gene Codes Corporation, Ann Arbor, USA) and checked manually. A total length of 763 bp of ND4 sequence was obtained from each individual.

\section{Genetic analyses}

Median joining networks were constructed using DNA Alignment ver. 1.0.0.3 and Network ver. 4.1.0.8 http:// www.fluxus-technology.com[70]. Genetic differentiation between pairs of populations ( $F_{\mathrm{ST}}$ values) was estimated from the sequence data using analysis of molecular variance (AMOVA) in ARLEQUIN version 3.01 [71]. Significance was estimated by 1000 permuations of the haplotypes between the populations.

\section{Cloning and sequencing of PCR products}

PCR products of the ND4 gene from individuals that yielded a mixed clade 1 and clade 2 sequence were cloned using the pGEM-T Vector System II kit according to the manufacturer's instruction (Promega Corporation, Madison, WI, USA). The same PCR primers were used to amplify the cloned ND4 gene fragments from colonies containing inserts. Colony material was prepared for PCR by heating a small portion of an individual colony in 20 $\mu \mathrm{l} \mathrm{dH_{2 }} \mathrm{O}$ to $95^{\circ} \mathrm{C}$ for 5 mins and $0.5 \mu \mathrm{l}$ of this was used in PCR reaction volumes of $25 \mu$ l. The amplifications were performed using the same reactant concentrations as above. The PCR conditions were initial denaturation at $94^{\circ} \mathrm{C}$ for 5 mins followed by 30 cycles of $92^{\circ} \mathrm{C}$ for $30 \mathrm{sec}$, $52^{\circ} \mathrm{C}$ for $1 \mathrm{~min}$ and $72^{\circ} \mathrm{C}$ for $40 \mathrm{sec}$, with a final extension at $72^{\circ} \mathrm{C}$ for 7 mins. The PCR products were purified and sequenced and the same procedures as outlined above were used to check sequences and to construct a median joining network.

\section{Fresh mtDNA separation and PCR amplification}

Laboratory-reared freshly killed Ae. aegypti mosquitoes originating from Thailand were used for the isolation and extraction of enriched mtDNA using an alkaline lysis method [72]. For each individual, DNA was extracted using a standard phenol/chloroform DNA extraction method [68] from both the starting homogenate (which contained both nuclear and mtDNA) and from the final mtDNA-rich supernatant. PCR amplification and sequencing of the ND4 region was carried out as above.

\section{Additional PCR reactions}

Primers designed and used for the specific amplification of clade 2 ND4 gene sequences and for the amplification of Numt junctions are given in Table 2 (see main text for details of their application). PCR was performed as before with the exception that no hot start was used and that the annealing temperature was increased to $55^{\circ} \mathrm{C}$ to achieve high specificity.

\section{Authors' contributions}

TH carried out the molecular genetic studies, data analysis, some of the fieldwork and drafted the manuscript. CW conceived the study, led its design and co-ordination and helped to draft the manuscript. WTL, PS, DS and MSC participated in study design and co-ordination. TS, SM, and PS conducted fieldwork to collect mosquito specimens. All authors read and approved the final manuscript.

\section{Acknowledgements}

We are grateful to Mr. Sein Thaung (DMR-LM, Yangon, Myanmar), and all the entomology field staff from Thailand, Myanmar and Cambodia for their invaluable assistance and help in the field mosquito sample collections. We also thank Douda Bensasson and Casey Bergman (University of Manchester) for helpful discussions. This study was funded by the Special Programme for Research and Training in Tropical Diseases, World Health Organization (WHO/TDR) Collaborative Research Project Grant IDA40198 and Research Training Grant (RTG) ID-A60987.

\section{References}

I. Brown MW: Polymorphism in mitochondrial DNA of humans as revealed by restriction endonuclease analysis. Proceedings of the National Academy of Sciences of the United States of America 1980, 77(6):3605-3609.

2. Hale L, Singh R: Mitochondrial DNA variation and genetic structure in populations of Drosophila melanogaster. Molecular Biology Evolution 1987, 4(6):622-637.

3. Avise J, Ball R, Arnold J: Current versus historical population sizes in vertebrate species with high gene flow: a comparison 
based on mitochondrial DNA lineages and inbreeding theory for neutral mutations. Mol Biol Evol 1988, 5(4):33I-344.

4. Guillen AKZ, Barrett GM, Takenaka O: Genetic diversity among African great apes based on mitochondrial DNA sequences. Biodiversity and Conservation 2005, I 4(9):222I-2233.

5. Avise JC, Arnold J, Ball RM, Bermingham E, Lamb T, Neigel JE, Reeb CA, Saunders NC: Intraspecific phylogeography: the mitochondrial DNA bridge between population genetics and systematics. Annual Review of Ecology and Systematics 1987, I 8(1):489-522.

6. Meixner M, Arias M, Sheppard W: Mitochondrial DNA polymorphisms in honey bee subspecies from Kenya. Apidologie 2000, $3 \mathrm{I}: 18 \mathrm{I}-190$

7. Shoemaker DD, Ahrens ME, Ross KG: Molecular phylogeny of fire ants of the Solenopsis saevissima species-group based on mtDNA sequences. Molecular Phylogenetics and Evolution 2006, 38(I):200-2I5.

8. Michel AP, Ingrasci MJ, Schemerhorn BJ, Kern M, Goff G, Coetzee M, Elissa N, Fontenille D, Vulule J, Lehmann T, et al.: Rangewide population genetic structure of the African malaria vector Anopheles funestus. Molecular Ecology 2005, I 4( I 4):4235-4248.

9. Besansky NJ, Lehmann T, Fahey GT, Fontenille D, Braack LEO, Hawley WA, Collins FH: Patterns of mitochondrial variation within and between African malaria vectors, Anopheles gambiae and Anopheles arabiensis, suggest extensive gene flow. Genetics 1997, |47(4): |817-1828.

10. Walton C, Handley JM, Tun-Lin W, Collins FH, Harbach RE, Baimai V, Butlin RK: Population structure and population history of Anopheles dirus mosquitoes in Southeast Asia. Molecular Biology and Evolution 2000, I7(6):962-974.

II. Birungi J, Munstermann LE: Genetic structure of Aedes albopictus (Diptera: Culicidae) populations based on mitochondrial ND5 sequences: evidence for an independent invasion into Brazil and United States. Annals of the Entomological Society of America 2002, 95: I25-132.

12. Cook S, Diallo M, Sall AA, Cooper A, Holmes EC: Mitochondrial markers for molecular identification of Aedes mosquitoes (Diptera: Culicidae) involved in transmission of arboviral disease in West Africa. Journal of Medical Entomology 2005, 42:19-28.

13. Remme JHF, Blas E, Chitsulo L, Desjeux PMP, Engers HD, Kanyok TP, Kayondo JFK, Kioy DW, Kumaraswami V, Lazdins JK, et al.: Strategic emphases for tropical diseases research: a TDR perspective. Trends in Parasitology 2002, I 8( I 0):42 I-426.

14. WHO: Basic and strategic research, molecular entomology committee workplan. 2004 [http://www.who.int/tdr/disease/den gue/direction.htm]

15. Hebert PDN, Cywinska A, Ball SL, deWaard JR: Biological identifications through DNA barcodes. PLoS Biology 2003, 270(I5 I 2):3|3-32|

16. Bazin E, Glemin S, Galtier N: Population size does not influence mitochondrial genetic diversity in animals. Science 2006 3 I 2(5773):570-572.

17. Ballard JWO, Whitlock MC: The incomplete natural history of mitochondria. Molecular Ecology 2004, 13(4):729-744.

18. Hurst GDD, Jiggins FM: Problems with mitochondrial DNA as a marker in population, phylogeographic and phylogenetic studies: the effects of inherited symbionts. PLoS Biology 2005, 272(1572): I525-I534.

19. Lopez JV, Yuhki N, Masuda R, Modi W, O'Brien SJ: Numt, a recent transfer and tandem amplification of mitochondrial DNA to the nuclear genome of the domestic cat. Journal of Molecular Evolution 1994, 39(2): 174-190.

20. Zhang $D-X$, Hewitt G: Nuclear integrations: challenges for mitochondrial DNA markers. Trends in Ecology and Evolution | 1996, II:247-25I.

21. Sorenson M, Quinn T: Numts: a challenge for avian systematics and population biology. The Auk 1998, II5:214-221.

22. Bensasson D, Zhang D-X, Hartl DL, Hewitt GM: Mitochondrial pseudogenes: evolution's misplaced witnesses. Trends in Ecology \& Evolution 200I, I6(6):3|4-32I.

23. Arctander P, Fjeldsa J: Andean tapaculos of the genus Scytalopus (Aves, Rhinocryptidae); a study of speciation using DNA sequence data. Experientia Basel Supplementum 1994, 68:205-225.

24. Arctander P: Comparison of a mitochondrial gene and a corresponding nuclear pseudogene. Proceedings of the Royal Society of London B 1995, 262:13-19.
25. Jensen-Seaman MI, Esteban E Sarmiento, Amos S Deinard, Kenneth K Kidd: Nuclear integrations of mitochondrial DNA in gorillas. American Journal of Primatology 2004, 63(3): 139-147.

26. Thalmann O, Hebler J, Poinar HN, Paabo S, Vigilant L: Unreliable mtDNA data due to nuclear insertions: a cautionary tale from analysis of humans and other great apes. Molecular Ecology 2004, I3(2):32I-335.

27. Thalmann O, Serre D, Hofreiter M, Lukas D, Eriksson J, Vigilant L: Nuclear insertions help and hinder inference of the evolutionary history of gorilla mtDNA. Molecular Ecology 2005 , I4(1): 179-188.

28. Antunes A, Pontius J, Ramos MJ, O'Brien SJ, Johnson WE: Mitochondrial Introgressions into the nuclear genome of the domestic cat. J Hered 2007, 98(5):4|4-420.

29. Pereira S, Baker A: Low number of mitochondrial pseudogenes in the chicken (Gallus gallus) nuclear genome: implications for molecular inference of population history and phylogenetics. BMC Evolutionary Biology 2004, 4(I): 17.

30. Pamilo P, Viljakainen L, Vihavainen A: Exceptionally high density of Numts in the honeybee genome. Molecular Biology and Evolution 2007, 24(6): 1340-1346.

3I. Martins J, Solomon SE, Mikheyev AS, Mueller UG, Ortiz A, Bacci M: Nuclear mitochondrial-like sequences in ants: evidence from Atta cephalotes (Formicidae: Attini). Insect Molecular Biology 2007, 16(6):777-784.

32. Richly E, Leister D: Numts in sequenced eukaryotic genomes. Molecular Biology and Evolution 2004, 2 I: I08I-1084.

33. Bensasson D, Petrov DA, Zhang D-X, Hartl DL, Hewitt GM: Genomic gigantism: DNA loss is slow in mountain grasshoppers. Mol Biol Evol 200 I, I 8(2):246-253

34. Nene V, Wortman IR, Lawson D, Haas B, Kodira C, Tu Z], Loftus B Xi Z, Megy K, Grabherr M, et al:: Genome Sequence of Aedes aegypti, a Major Arbovirus Vector. Science 2007, 316(5832): 17|8-1723.

35. Woischnik M, Moraes C: Pattern of organization of human mitochondrial pseudogenes in the nuclear genome. Genome Research 2002, I 2(6):885-893.

36. Tourmen Y, Baris O, Dessen P, Jacques C, Malthiery Y, Reynier P: Structure and chromosomal distribution of human mitochondrial pseudogenes. Genomics 2002, 80(I):7I-77.

37. Gorrochotegui-Escalante N, Gomez-Machorro C, Lozano-Fuentes $S$, Fernandez-Salas I, Munoz MD, Farfan-Ale JA, Garcia-Rejon J, Beaty B], Black WC: Breeding structure of Aedes aegypti populations in Mexico varies by region. American Journal of Tropical Medicine and Hygiene 2002, 66(2):213-222.

38. Bosio CF, Harrington LC, Jones JW, Sithiprasasna R, Norris DE, Scott TW: Genetic structure of Aedes aegypti populations in Thailand using mitochondrial DNA. American Journal of Tropical Medicine and Hygiene 2005, 72(4):434-442.

39. Costa-da-Silva AL, Capurro ML, Bracco JE: Genetic lineages in the yellow fever mosquito Aedes aegypti (Diptera: Culicidae) from Peru. Mem Inst Oswaldo Cruz, Rio de Janeiro 2005, 100(6):639-644.

40. Bracco JE, Capurro ML, Lourenco-de-Oliveira R, Sallum MAM: Genetic variability of Aedes aegypti in the Americas using a mitochondrial gene: evidence of multiple introductions. Mem Inst Oswaldo Cruz, Rio de Janeiro 2007, I 02(5):. ISSN 0074-0276

41. Herrera F, Urdaneta L, Rivero J, Zoghbi N, Ruiz J, Carrasquel G, Martínez JA, Pernalete M, Villegas P, Montoya A, et al.: Population genetic structure of the dengue mosquito Aedes aegypti in Venezuela. Memórias do Instituto Oswaldo Cruz, Rio de Janeiro 2006, 101:625-633.

42. Smith CEG: The history of dengue in tropical Asia and its probable relationship to the mosquito Aedes aegypti. Journal of Tropical Medicine and Hygiene 1956, 59:243-25I.

43. Tabachnick WJ, Powell JR: Worldwide survey of genetic variation in the yellow fever mosquito, Aedes aegypti. Genetical Research 1979, 34(3):215-229.

44. Tien TK, Vazeille-Falcoz M, Mousson L, Hoang TH, Rodhain F, Huong NT, Failloux AB: Aedes aegypti in Ho Chi Minh City (Viet Nam): susceptibility to dengue 2 virus and genetic differentiation. Transactions of the Royal Society of Tropical Medicine and Hygiene 1999, 93(6):58|-586.

45. Mousson L, Vazeille M, Chawprom S, Prajakwong S, Rodhain F, Failloux $A B$ : Genetic structure of Aedes aegypti populations in 
Chiang Mai (Thailand) and relation with dengue transmission. Tropical Medicine \& International Health 2002, 7(1 0):865-872.

46. Failloux $A B$, Vazeille $M$, Rodhain $F$ : Geographic genetic variation in populations of the dengue virus vector Aedes aegypti. Journal of Molecular Evolution 2002, 55(6):653-663.

47. Paduan KDS, Ribolla PEM: Mitochondrial DNA polymorphism and heteroplasmy in populations of Aedes aegypti in Brazil. Journal of Medical Entomology 2008:59-67.

48. Parr R, Maki J, Reguly B, Dakubo G, Aguirre A, Wittock R, Robinson $K$, Jakupciak J, Thayer R: The pseudo-mitochondrial genome influences mistakes in heteroplasmy interpretation. $B M C$ Genomics 2006, 7(I): 185.

49. Gorrochotegui-Escalante N, Munoz MD, Fernandez-Salas I, Beaty BJ, Black WC: Genetic isolation by distance among Aedes aegypt populations along the northeastern coast of Mexico. American Journal of Tropical Medicine and Hygiene 2000, 62(2):200-209.

50. Beebe NW, Whelan PI, Hurk Avd, Ritchie SA, Cooper RD: Genetic diversity of the dengue vector Aedes aegypti in Australia and implications for future surveillance and mainland incursion monitoring. Commun Dis Intell 2005, 29(3):299-304.

51. Haag-Liautard C, Coffey N, Houle D, Lynch M, Charlesworth B, Keightley PD: Direct estimation of the mitochondrial DNA mutation rate in Drosophila melanogaster. PLoS Biology 2008 6(8):e204

52. Kobayashi N, Tamura K, Aotsuka T: PCR error and molecular population genetics. Biochemical Genetics 1999 , 37(9II0):317-32I.

53. Triant D, DeWoody J: Molecular analyses of mitochondrial pseudogenes within the nuclear genome of arvicoline rodents. Genetica 2008, I32(1):21-33.

54. Ricchetti M, Tekaia F, Dujon B: Continued colonization of the human genome by mitochondrial DNA. PLOS Biology 2004, 2(9): $1313-1323$

55. Hazkani-Covo E, Graur D: A comparative analysis of Numt evolution in human and chimpazee. Molecular Biology and Evolution 2007, 24(I):13-18.

56. Urdaneta-Marquez L, Bosio C, Herrera F, Rubio-Palis Y, Salasek M, Black W IV: Genetic relationships among Aedes aegypti collections in Venezuela as determined by mitochondrial DNA variation and nuclear single nucleotide polymorphisms. American Journal of Tropical Medicine and Hygiene 2008, 78(3):479-49I.

57. Sorenson M, Fleischer R: Multiple independent transpositions of mitochondrial DNA control region sequences to the nucleus. Proceedings of the National Academy of Sciences of the United States of America 1996, 93:1 15239-15243.

58. Brower AV: Rapid morphological radiation and convergence among races of the butterfly Heliconius erato inferred from patterns of mitochondrial DNA evolution. Proceedings of the National Academy of Sciences of the United States of America 1994, 9 I( I4):649I-6495.

59. Paupy C, Brengues C, Kamgang B, Herve J-P, Fontenille D, Simard F: Gene flow between domestic and sylvan populations of Aedes aegypti (Diptera: Culicidae) in North Cameroon. Journal of Medical Entomology 2008, 45(3):391-400.

60. Huber K, Le Loan L, Chantha N, Failloux AB: Human transportation influences Aedes aegypti gene flow in Southeast Asia. Acta Tropica 2004, 90(1):23-29.

61. Ravel S, Monteny N, Olmos DV, Verdugo JE, Cuny G: A preliminary study of the population genetics of Aedes aegypti (Diptera: Culicidae) from Mexico using microsatellite and AFLP markers. Acta Tropica 200I, 78(3):24I-250.

62. Song $\mathrm{H}$, Buhay JE, Whiting MF, Crandall KA: Many species in one: DNA barcoding overestimates the number of species when nuclear mitochondrial pseudogenes are coamplified. Proceedings of the National Academy of Sciences 2008, 105(36): I3486- 1349I.

63. Altschul S, Gish W, Miller W, Myers E, Lipman D: Basic local alignment search tool. J Mol Biol 1990, 2 I 5(3):403-4I0.

64. Behura S: Analysis of nuclear copies of mitochondrial sequences in honey bee Apis mellifera genome. Mol Biol and Evol 2007, 24(7): 1492-1505.

65. Thompson JD, Gibson TJ, Plewniak F, Jeanmougin F, Higgins DG: The ClustalX windows interface: flexible strategies for multiple sequence alignment aided by quality analysis tools. Nucleic Acids Research 1997, 25:4876-4882.
66. Jeanmougin F, Thompson J, Gouy M, Higgins DG, Gibson TJ: Multiple sequence alignment with Clustal $\mathbf{X}$. Trends in Biochemical Sciences 1998, 23:403-405.

67. Tamura K, Dudley J, Nei M, Kumar S: MEGA4: Molecular evolutionary genetics analysis (MEGA) software version 4.0. Molecular Biology and Evolution 2007, 24:1596-1599.

68. Sambrook J, Russell DW: Molecular cloning: a laboratory manual. 3rd edition. New York: Cold Spring Harbor Laboratory Press; 2001 .

69. Rozen S, Skaletsky H: Primer3 on the WWW for General Users and for Biologist Programmers. In Methods in Molecular Biology, Bioinformatics Methods and Protocols Volume 132. Edited by: Misener S, Krawetz SA. Totowa, NJ: Humana Press Inc; 1999:365-386.

70. Bandelt $H J$, Forster $P$, Rohl A: Median-joining networks for inferring intraspecific phylogenies. Mol Biol Evol 1999, I6(I):37-48.

7I. Excoffier L, Smouse PE, Quattro JM: Analysis of molecular variance inferred from metric distances among DNA haplotypes - application to human mitochondrial DNA restriction data. Genetics 1992, I31(2):479-49I.

72. Tamura K, Aotsuka T: Rapid isolation method of animal mitochondrial DNA by the alkaline lysis procedure. Biochemical Genetics 1988, 26(II):8I5-8I9.
Publish with BioMed Central and every scientist can read your work free of charge

"BioMed Central will be the most significant development for disseminating the results of biomedical research in our lifetime. "

Sir Paul Nurse, Cancer Research UK

Your research papers will be:

- available free of charge to the entire biomedical community

- peer reviewed and published immediately upon acceptance

- cited in PubMed and archived on PubMed Central

- yours - you keep the copyright

Submit your manuscript here:

http://www.biomedcentral.com/info/publishing_adv.asp
BioMedcentral 